\title{
Impact of Selfish Packet Forwarding on Energy-Efficient Topology Control
}

\author{
Ramakant S. Komali and Allen B. MacKenzie \\ Virginia Polytechnic Institute and State University; Blacksburg, Virginia 24061 \\ Email: $\{$ rskomali, mackenab\}@vt.edu
}

\begin{abstract}
Wireless topology control attempts to harness the programmability of radios to build efficient network structures. This paper addresses energy minimization in an ad hoc network of selfish nodes by jointly considering packet forwarding and packet sourcing-two main sources of energy consumption in multi-hop networks. Specifically, we examine how energy-efficient topologies can be established through non-cooperative power control taking into account the possibility of selective and partial packet forwarding in the network.

The topology control game, where each node is attempting to minimize its energy consumption while maintaining network connectivity, is shown to be a potential game. This establishes the existence of a Nash Equilibrium (NE). Additionally, the $\mathrm{NE}$ is shown to be unique and globally energy-efficient-the nodes transmit at just enough power necessary to maintain all the minimum energy routes in the network. From simulations we observe that when nodes forward a small percentage of packets directed through them, the resulting $\mathrm{NE}$ topologies that minimize energy are more densely connected and consume more energy than the topologies that emerge when nodes forward a large portion of incoming packets. From the energy viewpoint, this result is particularly interesting as it quantifies the energy efficiency gains obtained by cooperation and corroborates the need for encouraging nodes to forward packets in a decentralized network.
\end{abstract}

\section{INTRODUCTION}

Ad hoc networks hold promise not only because of their immense potential to aid military applications, emergency disaster rescue-and-relief operations, pervasive computing, automotive networks, and home networking, but also because of their ease and speed of deployment. These networks can be established on-the-fly when heterogeneous devices (such as PDAs, laptops, cellular phones etc.)—distributed over a geographical region-communicate with each other wirelessly, in a multi-hop manner without any "fixed" infrastructure. With the devices moving around the network or changing their transmission parameters, the resulting dynamic network topology needs to be efficiently managed. This, coupled with the vulnerability and limitations of the wireless medium, poses a significant challenge to efficient network design.

Ad hoc network nodes are typically portable and are equipped with radios, memory, and processors, all powered by a battery. Hence, it is imperative that the protocols be energy-efficient; this not only increases the operational lifetime of the network, but also contributes to better network performance. Designing energy-efficient algorithms is thus

This work is supported by National Science Foundation (NSF) grant CNS0448131 . of paramount importance in ad hoc networks [1]. Topology Control - the study of how to assign transmission parameters so as to achieve certain network-wide goals-is one such design technique used to enhance global network performance. Topology Control (TC) algorithms strive to deliver energyefficient networks while achieving certain end-to-end network objectives (such as minimizing latency, maximizing lifetime etc.).

Ad hoc networks are expected to be fully distributed and controlled by end-users. From a user's perspective, nodes are either competing for network resources (e.g. bandwidth) or conserving their own limited resources (e.g. battery energy). Given that modern radios are increasingly autonomous and reconfigurable, it seems likely that nodes may behave in way so as to conserve their resources and act in their self-interest.

In this work, we address the issues of energy minimization, topology control, and selfish node behavior. From a topology control perspective, nodes consume energy in transmitting their own packets as well as in forwarding packets for other nodes. In this sense, nodes may selfishly minimize their energy consumption by limiting not only their transmit power levels but also by regulating the portion of packets which they forward for others. In literature, these two issues-power control and packet forwarding-have largely been studied in isolation. For instance, [2], [3] examine the impact of selfish power control on steady state topologies; likewise, [4], [5] analyze the role of selfish packet forwarding on network performance. Here, we consider the effect of packet forwarding levels ${ }^{1}$ on power control for energy-efficient topology design. In particular, our work addresses two questions related to distributed topology control: in selecting their operating parameters, should nodes be programmed to optimize their own "selfish" objectives, or a network-wide objective function?; and, how much packet forwarding is necessary to make a decentralized selfish network energy-optimal?

We develop a game-theoretic framework for studying the above problems in the context of energy minimization. Under this framework, we propose a notion of expected energy consumption to characterize the energy consumed by routes containing nodes that selectively forward packets with a certain probability. The Topology Control Game (TCG),

\footnotetext{
${ }^{1}$ The term "level" is taken to mean the fraction of packets that nodes forward among those that are directed through them. We measure this level as a probability and sometimes use the terms probability and level interchangeably in this manuscript.
} 
with each node controlling its transmit power to minimize its energy consumption locally while maintaining network connectivity, is shown to be a potential game. Potential games (over compact action spaces) automatically guarantee the existence of at least one pure strategy NE [6]. We show that the TCG admits a unique NE. We also establish that the NE is globally efficient, i.e., given the forward levels, it minimizes the aggregate energy consumed by the network by preserving the minimum energy paths between every node pair. We propose a simple best response (BR) algorithm for the selfish nodes, to ensure convergence to these energyefficient states. Using simulations, we characterize the NE topologies for various packet forwarding levels. Specifically, we observe that at higher forwarding levels, the resulting topologies that minimize energy get sparser, and the average path length increases. We also observe that the NE topologies containing low forwarding nodes consume much more energy than those containing nodes that forward a higher fraction of packets directed through them. This result substantiates the need for incentivizing selfish nodes to cooperate and forward packets in energy-limited networks. Note that, incentivizing and stimulating nodes to forward packets by means of a reputation-based mechanism often involves large overhead costs. Using our analysis on network performance in partially forwarding networks and accounting for the overhead costs associated with stimulating node cooperation, an optimum level of forwarding can be determined as a design decision.

The remainder of this paper is organized as follows: we begin by providing a brief overview of game theory as applicable to our work in Section II. We discuss the system model, framework and assumptions in Section III. We then analyze the TCG and discuss its implications in the context of energy efficiency in Section IV. We present simulation results in Section V. We review the related work on non-cooperative topology control in Section VI. We conclude in Section VII.

\section{Game Theory and Potential Games}

In this section, we present a brief overview of important elements and notations of non-cooperative, strategic form game theory. For a rigorous treatment of these and other topics in game theory, refer to [7]. Here, we specifically focus on potential games.

The main object of game-theoretic study is the game, which is a formal model of an interactive decision-making situation. A strategic non-cooperative game $\Gamma=\left\langle N, A, u_{i}\right\rangle$ has three components:

1) Player set $N: N=\{1,2, \ldots, n\}$ where $n$ is the number of players in the game.

2) Action set $A: a \in A=\times_{i=1}^{n} A_{i}$ is the space of all action vectors (tuple), where each component, $a_{i}$, of the vector $a$ belongs to the set $A_{i}$, the set of actions of player $i$. Often we denote action profile $a=\left(a_{i}, a_{-i}\right)$ where $a_{i}$ is player $i$ 's action and $a_{-i}$ denotes the actions of the other $n-1$ players. Similarly, $A_{-i}=\times_{j \neq i} A_{j}$ is used to denote the set of action profiles for all players except $i$.
3) For each player $i \in N$, utility function $u_{i}: A \rightarrow \mathbb{R}$, models players' preferences over action profiles. $u=$ $\left(u_{1}, \ldots, u_{n}\right): A \rightarrow \mathbb{R}^{n}$ denotes the vector of such utility functions.

The most common solution concept for such games is the Nash Equilibrium, which, in some sense, is a consistent predictor of possible outcomes of a game. A NE is a stable point because no player has any incentive to unilaterally change his action from it.

Definition An action profile $a^{*}=\left(a_{i}^{*}, a_{-i}^{*}\right)$ is a Nash Equilibrium if, $\forall i \in N$ and $\forall a_{i} \in A_{i}$,

$$
u_{i}\left(a^{*}\right) \geq u_{i}\left(a_{i}, a_{-i}^{*}\right)
$$

A game may possess a large number of Nash equilibria or none at all. Some classes of games are known to possess at least one NE.

Definition A strategic game $\Gamma=\left\langle N, A, u_{i}\right\rangle$ is an Ordinal Potential Game (OPG) if there exists a function $V: A \rightarrow \mathbb{R}$ such that $\forall i \in N, \forall a_{-i} \in A_{-i}$, and $\forall a_{i}, b_{i} \in A_{i}$

$V\left(a_{i}, a_{-i}\right)-V\left(b_{i}, a_{-i}\right)>0 \Leftrightarrow u_{i}\left(a_{i}, a_{-i}\right)-u_{i}\left(b_{i}, a_{-i}\right)>0$

$V$ is called the Ordinal Potential Function (OPF) of $\Gamma$.

In essence, an OPG requires payoffs that exhibit the same "directional" behavior, when that individual unilaterally deviates.

Potential games with compact action spaces are known to possess at least one NE in pure strategies [6]. The following lemma due to [6] establishes how Nash equilibria of the game can be identified.

Lemma 1: Let $\Gamma$ be an OPG and $V$ its corresponding OPF. If $a \in A$ maximizes $V$, then it is a NE.

Thus, potential maximizers form a subset of the NE of a potential game. If we can identify potential functions for a game, we can immediately identify some NE of the game by solving for the potential maximizers. Potential games also exhibit certain convergence properties that are often useful when dealing with algorithmic analysis. Rather than delving into these technical details, we simply refer to [6] and [8] for a primer.

\section{System Model AND Assumptions}

\section{A. Network Model}

We model the network as consisting of nodes equipped with omnidirectional antennas with isotropic transmission patterns; the transmission range is modeled as a disc. The topology created by the connections is modeled as a communcation graph $G=(N, E)$ where $N$ is the set of nodes and $E$ is a set of directed arcs representing unidirectional connections. (Equation (3) shows the set of connections: a connection exists if the transmission power $\left(p_{i}\right)$ is greater than the thermal noise $\left(\sigma_{j}\right)$ and Signal to Noise Ratio (SNR) requirement $\left(\gamma_{j}\right)$ at the receiving radio, given the gain (loss) factor between the two (contained in the matrix $\left.\left[g_{i j}\right]\right)$ ). We only consider thermal 
noise and disregard interference by assuming the existence of a MAC protocol to de-conflict transmissions.

$$
E=\left\{\overrightarrow{i j} \mid \frac{p_{i} g_{i j}}{\sigma_{j}} \geq \gamma_{j}\right\}
$$

The term $\sigma_{j} \gamma_{j} / g_{i j}$ will be referred to as $\omega(i, j)$, which is the threshold transmission power required to close a connection from radio $i$ to radio $j$. As the exact threshold $\omega(i, j)$ is a function of channel attenuation and inter-nodal separation, our model is quite generalized to accomodate varying channel characteristics. Once $\omega$ is specified, the condition $p_{i} \geq \omega(i, j)$ determines all the feasible transmissions. The induced topology $G$ is connected if and only if there exists a path-a collection of contiguous edges from $E$-between every node pair in $N$. In a slight abuse of notation, we will sometimes use $\mathbf{p}$ to represent $G$ (noting that $\mathbf{p}$ induces $G$ ). We also represent by $G_{\max }$ the topologies induced when all nodes transmit at their maximum power level $p_{i}^{\max }$.

\section{B. Energy Model}

In order to select an appropriate power level for data transmission, nodes utilize the per-packet power control approach. Appropriating powers on a per-packet basis has been shown to be an effective power control strategy in networks where nodes are non-homogeneously scattered [9]. Additionally, several variants of 802.11 MAC protocols that use per-packet power control have been proposed, e.g., PCM [10], MACA [11], PCMA [12]. The basic idea is that RTS-CTS packets are sent at the highest power levels whereas DATA-ACK packets are transmitted at much lower power levels (see [13]). A similar scheme is employed in [14], where each node maintains a table that stores the minimum transmit powers needed to reach each of its neighbors. In all these schemes, the use of perpacket technique is shown to be effective in reducing energy consumption and improving network throughput. Besides, minimum energy routing based on the power threshold metric is also common among routing protocols, e.g., PARO [13].

In addition to data transmission energy costs, nodes also consume energy due to all additional overhead traffic such as periodic exchange of Hello and TOPOLOGY CONTROL messages. We assume that control traffic makes up a significant portion of total traffic in the network and therefore cannot be neglected. This is particularly true in ad-hoc networks where network conditions are dynamic due to the frequent disruptions and link instabilities. Similar to the RTS-CTS philosophy, we assume that these control messages are transmitted at the highest power level necessary for a node to communicate with its "farthest" neighbor. The rationale behind such an assumption is evident: TOPOLOGY CONTROL messages are typically transmitted as broadcast packets that are usually exchanged by neighbors to collect information about any changes in the topology. Given this, control energy consumed by a node becomes a function of its power level $p_{i}$; we represent such energy costs by $\mathcal{E}_{i}^{c}\left(p_{i}\right)$. For the purpose of our analysis, we only require that $\mathcal{E}_{i}^{c}$ be a monontonic function.

Using the per-packet model of data transmission, we now derive an expression for the energy consumed by a node in transmittng data packets to its destination. In all subsequent discussions, we base our analysis on the assumption that all transmissions are unicast. Given that the traffic flows between all source-destination pairs may traverse multiple hops, intermediate nodes may choose to forward only a portion of the packets directed through them. We assume that nodes do not differentiate between flows when forwarding; this allows us to represent the fraction of traffic forwarded by an intermediate node $i$ simply as a probability $q_{i}$. Thus, nodes randomly drop packets of other flows through them according to its chosen $q_{i}$.

To examine energy-efficient topologies when nodes forward packets sporadically, i.e., the forward levels $q_{i} \in(0,1]$, it makes sense to consider the expected energy consumption metric. Consider a path $\mathcal{S}_{i j}$ containing a set of intermediate nodes between source $i$ and destination $j$; without loss of generality, let the node ids be ordered, i.e., $\mathcal{S}_{i j}=(i, i+1, \ldots, j-1, j)$. Then, the expected energy consumed in transporting messages from $i$ to $j$ along $\mathcal{S}_{i j}$ is given by:

$$
\mathcal{E}_{i j}=\sum_{\substack{k \in \mathcal{S}_{i j} \\ k \neq j}} \overline{\mathcal{E}}_{k}
$$

Here, $\overline{\mathcal{E}}_{k}$ is the expected energy consumed by node $k$. To determine $\overline{\mathcal{E}}_{k}$, we suppose that packets that are dropped at a certain node are retransmitted by its previous hop node until they are successfully forwarded $\mathrm{on}^{2}$. This assumption parallels the strategies used in medium access transmission schemes that are often used in literature. We believe that triggering a retransmission due to packet drops can easily be implemented at the MAC without much modification to it; this is because, the packet drops due to selfish behavior are functionally similar to the packet losses due to channel errors. The link-level retransmission strategy is also well-motivated from an energy efficiency standpoint [15]. Due to the additive construct of (4), the model also displays a distributed structure: the energy of each segment can be separately evaluated and then summed up.

We derive an expression for $\overline{\mathcal{E}}_{k}$, by calculating the expected number of transmissions at $k$. Because $k$ retransmits only when its next hop node drops packets, it is sufficient to consider a two-hop path, as shown in Figure 1, to calculate $\overline{\mathcal{E}}_{k}$. For

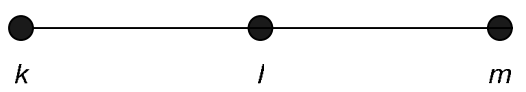

Fig. 1. An arbitrary route from $k$ to $m$ containing one intermediate node (l).

simplicity of analysis, we set packet transmission durations to

\footnotetext{
${ }^{2}$ Because we are studying the effect of selfish packet forwarding, we consider all other network conditions to be ideal. Therefore, packets are dropped only due to selfish node behaviour and not because of errors due to channel conditions, collisions due to contentions at the MAC layer, or due to congestion issues.
} 
1 time unit assuming that all data packets are of equal length. Thus, given that $l$ forwards with probability $q_{l}$, the expected energy consumed by $k$ can be obtained as:

$$
\overline{\mathcal{E}}_{k}=p_{k} E[T]
$$

where $p_{k}$ is the transmission power of node $k$, and $E[T]$ is the expected number of transmissions at $k$. In the above example, $p_{k}=\omega(k, l)$ under the per-packet transmission model. Since $T$ is a geometric random variable, the probability that we have $t$ transmissions at node $k$ is given by:

$$
P[t]=\left(1-q_{l}\right)^{t-1} q_{l}
$$

and $E[T]=\frac{1}{q_{l}}$.

Thus, the expected energy consumed by $k$ becomes,

$$
\overline{\mathcal{E}}_{k}=\omega(k, l) / q_{l}
$$

Combining (7) and (4), we obtain the expected energy consumed by an arbitrary route $\mathcal{S}_{i j}$ :

$$
\mathcal{E}_{i j}=\omega(j-1, j)+\sum_{\substack{k \in \mathcal{S}_{i j} \\ k<j-1}} \omega(k, k+1) / q_{k+1}
$$

As a special case, when $\mathbf{q}=\mathbf{1}$, the energy consumed by $\mathcal{S}_{i j}$ becomes:

$$
\mathcal{E}_{i j}=\sum_{\substack{k \in \mathcal{S}_{i j} \\ k \neq j}} \omega(k, k+1)
$$

$\mathcal{S}_{i j}$ is called a minimum energy path if it consumes the least amount of energy to transport packets from $i$ to $j$, among all such paths between $i$ and $j$. This minimum energy is given by:

$$
\mathcal{E}_{i j}^{\min }=\min _{\forall \mathcal{S}_{i j}} \mathcal{E}_{i j}
$$

An energy-efficient protocol is said to have the minimum energy property if it preserves the minimum energy paths between every source-destination pair [16]. Using this definition, the objective of our distributed topology control algorithm is to derive a subgraph $G_{\min }$ of $G_{\max }$ that has the minimum energy property. $G_{\min }$ is induced by a power vector which we denote by $\mathbf{p}^{\min }=\left(p_{1}^{\min }, \ldots, p_{n}^{\min }\right)$, where $n$ is the number of nodes in the networks.

Our model for energy consumption $\left(\mathcal{E}_{i}\right)$ takes into account the energy consumed in transmitting data packets $\left(\mathcal{E}_{i}^{d}\right)$ and in transmitting control packets $\left(\mathcal{E}_{i}^{c}\right)$. In the following section, we introduce the node utility function that specifies the exact contributions from these two components and, how $\mathcal{E}_{i}^{d}$ can be determined from (8) and (10).

\section{Game-Theoretic Model}

In our framework, each node takes a selfish view of minimizing its energy expenditure, possibly at the expense of other nodes', and even the network, performance. One way of modeling these interactions between selfish network nodes is as a non-cooperative game. Specifically, a topology control process can be viewed as normal form game: individual nodes form the player set, $N \equiv\{1,2, \ldots, n\}$, of the game.
Each node can autonomously set its transmit power level $p_{i} \in\left[0, p_{i}^{\max }\right]$. The individual power levels can be collected into a power vector $\mathbf{p}=\left(p_{1}, p_{2}, \ldots, p_{n}\right)$, which forms the action space, $A$, for the game. Each node perceives a trade-off between the benefit it derives from a connected topology and the cost incurred in establishing it. A utility function captures these tradeoffs and maps the power vector to a payoff for each node.

To enable multi-hop routing, the underlying topology must not only be connected, but also contain paths where intermediate nodes are willing to forward packets for others. In this work, we assume that packet forwarding levels, $q_{i}$, are selected exogenously. A node can decide to cooperate intermittently, forwarding packets for others with some probability $q_{i} \in$ $(0,1]$. The subject of how to spur nodes to cooperate and forward packets for others is a research thread in itself; we refer interested readers to [5], [17] (and references contained therein). Given the packet forwarding levels, we are interested in analyzing what topologies emerge in steady state when nodes optimize their performance with respect to connectivity and energy minimization goals.

Because of their energy constraint (let $\mathcal{E}_{i}^{\max }$ be the maximum energy of each node), each node faces a temptation to conserve its total energy $\mathcal{E}_{i}$ by reducing its power level and selecting only closeby neighbors for relaying transmissions. Additionally, because certain nodes forward packets with low probabilities, it may necessitate other nodes to increase their transmission power to circumvent these noncooperating nodes. It stands to reason that an appropriate power level selection depends not only on other nodes' power level but also on their packet forwarding levels. We cast these interdependencies in a utility function, which for each node is given by:

$$
u_{i}(\mathbf{p})=\alpha_{i} f_{i}(\mathbf{p})-\beta_{i} \mathcal{E}_{i}^{d}(\mathbf{p})-\kappa_{i} \mathcal{E}_{i}^{c}\left(p_{i}\right)
$$

where $f_{i}$ is the number of nodes that can be reached (possibly over multiple hops) by node $i$. The last two terms collectively represent $\mathcal{E}_{i}$, the total energy consumed by $i$. The terms $\beta_{i}$ and $\kappa_{i}$ are fractional scalars and are chosen such that no node will try to lower its control energy at the cost of increased data energy. Thus, no node reduces its power lower than that required to keep all its minimum energy routes. In some sense, $\beta_{i}$ is the dominant term and the total energy is dictated by the data energy term. Finally, by picking $\alpha_{i} \geq \mathcal{E}_{i}^{\max }$, we cast network connectivity as a constraint in our model.

Without a priori knowledge of traffic requirements, each node acts under the premise that all destinations are equally likely. This allows us to express the energy consumed for data transmissions as $\mathcal{E}_{i}^{d}(\mathbf{p})=\sum_{j \neq i} \mathcal{E}_{i j}^{\min }(\mathbf{p})$, where $\mathcal{E}_{i j}^{\min }$ is given by (10). Note that, even though (8) doesn't contain the $p_{i}$ terms explicitly, $\mathcal{E}_{i j}$ is specified only for the existing routes, which are defined by the power assignment vector $\mathbf{p}$. The expression for $\mathcal{E}_{i}^{d}$ implicitly suggests that nodes that initiate a packet forwarding request are responsible for the entire cost associated with transporting messages to the destination. 
This technique is similar to mechanism design approaches and credit/debit schemes used in energy-efficient topology design [18], [19]. Additionally, the model also allows us to reduce the complexity by decoupling the problems of regulating packet forwarding and power control for energy minimization ${ }^{3}$.

Henceforth, the above game-theoretic framework will be referred to as the TCG. In the following sections, we study the impact of packet forwarding levels on transmit power selections in the context of energy-efficient TC. In particular, we analyze what topologies emerge in a TCG, and how well they perform in terms of energy efficiency.

\section{Game-Theoretic Analysis}

We begin by identifying some useful properties that analyze the effect of power adaptations in the TCG $\Gamma=\left\langle N, A, u_{i}\right\rangle$, where the individual utilities are given by (11). For the sake of brevity, we represent $\omega(i, j)$ by a more compact notation $\omega_{i j}$ in the following discussions.

Lemma 2: Every node $i$ that lowers (increases) the energy of a path $\mathcal{S}_{i k}$ also lowers (increases) the energy of all its longer paths $\mathcal{S}_{i d} \supseteq \mathcal{S}_{i k}$.

Proof: The proof follows from the additive structure of (8). Without loss of generality, consider two paths $\mathcal{S}_{i k}=$ $\{i, j, k\}$ and $\mathcal{S}_{i k}^{\prime}=\{i, l, m, k\}$. Let $i$ lower $\mathcal{E}_{i k}$ by reducing its power from $p_{i}$ to $p_{i}^{\prime}$ to remove $\overrightarrow{i j}$, and selecting a different route $\mathcal{S}_{i k}^{\prime}$ instead. This means, $\omega_{i l} / q_{l}+\omega_{l m} / q_{m}+\omega_{m k}<$ $\omega_{i j} / q_{j}+\omega_{j k}$, implying, $\omega_{i l} / q_{l}+\omega_{l m} / q_{m}-\omega_{i j} / q_{j}<\omega_{j k}-$ $\omega_{m k} \leq\left(\omega_{j k}-\omega_{m k}\right) / q_{k}$. Rearranging the above inequality (and adding $\omega_{k d}$ to both sides) we get, $\omega_{i l} / q_{l}+\omega_{l m} / q_{m}+$ $\omega_{m k} / q_{k}+\omega_{k d}<\omega_{i j} / q_{j}+\omega_{j k} / q_{k}+\omega_{k d}$. This means, any other path going through $k, \mathcal{S}_{i d}^{\prime}=\{i, l, m, k, d\}$ also has lower energy than $\mathcal{S}_{i d}=\{i, j, k, d\}$.

Now, let $i$ increase $\mathcal{E}_{i k}$. Using the same argument as above, $\omega_{i j} / q_{j}+\omega_{j k}<\omega_{i l} / q_{l}+\omega_{l m} / q_{m}+\omega_{m k}$. This implies, $\omega_{i j} / q_{j}<$ $\omega_{i l} / q_{l}+\omega_{l m} / q_{m}+\omega_{m k}-\omega_{j k} \leq \omega_{i l} / q_{l}+\omega_{l m} / q_{m}+\omega_{m k} / q_{k}-$ $\omega_{j k} / q_{k}$. Rearranging the terms and adding $\omega_{k d}$ to both sides, we get $\omega_{i j} / q_{j}+\omega_{j k} / q_{k}+\omega_{k d}<\omega_{i l} / q_{l}+\omega_{l m} / q_{m}+\omega_{m k} / q_{k}+$ $\omega_{k d}$. This means, energy $\mathcal{E}_{i d}$ of path $\mathcal{S}_{i d} \supseteq \mathcal{S}_{i k}$ increases.

For the reverse direction of the above lemma, the following lemma does holds.

Lemma 3: Every node $i$ that increases the energy of a path $\mathcal{S}_{i d}$ also increases the energy of all its shorter paths $\mathcal{S}_{i k} \subseteq \mathcal{S}_{i d}$. We omit the proof, which is similar to the one given above.

Lemmas 2 and 3 underscore the coupling between the route costs $\mathcal{E}_{i j}$ associated with a particular node $i$. Lemma 4 specifies how a node's decision may impact another node's route cost.

Lemma 4: If node $i$ removes connections on a route $\mathcal{S}_{j k}$ and increases (decreases) $\mathcal{E}_{j k}$ for some node $j$, it also in-

\footnotetext{
${ }^{3}$ Under this energy model, it may seem counter-intuitive to consider the $\mathbf{q} \neq \mathbf{1}$ cases. However, because of the bandwidth limitations posed by adhoc networks, nodes may choose not to share such resources and therefore not forward packets. Note that forwarding levels are chosen exogenously; the rationale for choosing such levels in the context of energy minimization is a subject of future work. Interestingly, however, the steady state topologies under $\mathbf{q}=\mathbf{1}$ case consume the least energy among all other topologies that emerge under various q's (we show this in the next section).
}

creases (decreases) energy of its own route $\mathcal{S}_{i k} \subseteq \mathcal{S}_{j k}$ to $k$. Alternately, if node $i$ reduces (increases) its energy $\mathcal{E}_{i k}$ consumed on a route $\mathcal{S}_{i k}$, it also reduces (increases) the energy $\mathcal{E}_{j k}$ consumed on the route $\mathcal{S}_{j k} \supseteq \mathcal{S}_{i k}$ for some $j$.

Proof: Consider the case when $\mathbf{q}=1$ : For $i$ to be able to remove connections from a $\mathcal{S}_{j k}$ for $j, i$ must be an intermediate node on this route, i.e. $i \in \mathcal{S}_{j k}$. From the additive cost structure, it follows that if $\mathcal{S}_{j k}$ has the minimum $\mathcal{E}_{j k}$, then $\mathcal{S}_{i k} \subseteq \mathcal{S}_{j k}$ must also minimize $\mathcal{E}_{i k}$. Thus, when $i$ removes a connection on $\mathcal{S}_{j k}$ to increase $\mathcal{E}_{j k}, \mathcal{E}_{i k}$ also increases.

Now consider the case when $\mathbf{q} \neq 1$ : Without loss of generality, let $\mathcal{S}_{j k}=\{j, i, k\}$ be the minimum cost path from $j$ to $k$. Let there exist another path $\mathcal{S}_{i k}=\{i, m, k\}$ from $i$ to $k$. Let $i$ reduce its power from $p_{i}^{\prime}$ to $p_{i}$ to remove connection $\overrightarrow{i k}$ from $\mathcal{S}_{j k}$, and increase $\mathcal{E}_{j k}$. This implies, $\omega_{j i} / q_{i}+\omega_{i m} / q_{m}+\omega_{m k}>\omega_{j i} / q_{i}+\omega_{i k}$. In turn, this implies $\omega_{i m} / q_{m}+\omega_{m k}>\omega_{i k}$, meaning that $\mathcal{E}_{i k}$ also increases. For the decrease part the inequalities are reversed, and the result follows.

The following lemma quantifies the cause-effect relationship specified by Lemma 4 .

Lemma 5: If node $i$ changes the energy of a route $\mathcal{S}_{i k}$ by $\delta_{i}$, energy of route $\mathcal{S}_{j k} \supseteq \mathcal{S}_{i k}$ also changes by $\delta_{i}$.

Proof: Let $i$ increase (likewise, decrease) energy of a route $\mathcal{S}_{i k}$ from $\mathcal{E}_{i k}$ to $\mathcal{E}_{i k}^{\prime}=\mathcal{E}_{i k}+\delta_{i}$. From the additive structure of energy consumption, the original energy of a route, $\mathcal{S}_{j k}$, going through $i$, is given by: $\mathcal{E}_{j k}=\sum_{m \in \mathcal{S}_{j i} ; m \neq i} \overline{\mathcal{E}}_{m}+$ $\mathcal{E}_{i k}$. Therefore, $\mathcal{E}_{j k}^{\prime}=\mathcal{E}_{j k}+\delta_{i}$.

\section{A. Analysis of the TCG}

Having discussed the dynamics and implications of altering routes and changing routing costs, we now analyze the TCG $\Gamma$ and its NE outcomes. We first show that when a node unilaterally decreases (or increases) its utility, the utility of other nodes either remains unaffected or decreases (or increases). For the ease of exposition of the following results, we recast (11) as:

$$
u_{i}(\mathbf{p})=\alpha_{i} f_{i}(\mathbf{p})-\mathcal{E}_{i}(\mathbf{p})
$$

Theorem 6: The TCG $\Gamma$, where the individual utilities given by (11) and (12), is an OPG. The OPF is given by:

$$
V(\mathbf{p})=\sum_{i} u_{i}(\mathbf{p})
$$

Proof: We prove by applying the definition of OPGs. First,

$$
\begin{aligned}
\Delta V(\mathbf{p}) & =\sum_{j} \Delta u_{j}(\mathbf{p}) \\
& =\Delta u_{i}(\mathbf{p})+\sum_{j \neq i} \Delta u_{j}(\mathbf{p})
\end{aligned}
$$

- Consider the case $\Delta u_{i}(\mathbf{p})>0$. This implies $\Delta f_{i}(\mathbf{p})>0$ or $\Delta f_{i}(\mathbf{p})=0$ and $\Delta \mathcal{E}_{i}(\mathbf{p})<0$ (or both hold true). When the former holds, it is straightforward to see that $\Delta f_{j}(\mathbf{p}) \geq 0$ (equality holds when $j$ is not connected to $i$ ), which then implies that, for every $j, \Delta u_{j}(\mathbf{p}) \geq 0$. The latter case is more interesting to analyze. Observe that $\Delta f_{i}(\mathbf{p})=0 \Rightarrow \Delta f_{j}(\mathbf{p})=0, \forall j$. From our discussion on the choice of $\beta_{i}$ and $\kappa_{i}$ in Section III.C, $\Delta \mathcal{E}_{i}(\mathbf{p})<0 \Rightarrow \Delta \mathcal{E}_{i}^{d}(\mathbf{p}) \leq 0$, which in turn implies 
$\exists j$ for which $\mathcal{E}_{i j}^{\min }(\mathbf{p})$ is reduced. (This may occur if a new minimum energy route is created by $i$ by virtue of increasing its $p_{i}$. Adding a new route is also consistent with Lemma 2.) From Lemma 4, it follows that the energy of path $\mathcal{S}_{k j} \supseteq \mathcal{S}_{i j}, \mathcal{E}_{k j}^{\min }(\mathbf{p})$ either decreases or remains the same (if $k$ doesn't route through $i$ ). This implies, $\mathcal{E}_{j}^{d}(\mathbf{p})$ and therefore, $\mathcal{E}_{j}(\mathbf{p})$ doesn't increase. Thus, $\Delta \mathcal{E}_{i}(\mathbf{p})<0 \Rightarrow \Delta \mathcal{E}_{j}(\mathbf{p}) \leq 0 \forall j$. We have therefore shown that, $\Delta u_{i}(\mathbf{p})>0 \Rightarrow \Delta V(\mathbf{p})>0$.

- Now consider the case $\Delta u_{i}(\mathbf{p})<0$. In this case, $\Delta \mathcal{E}_{i}(\mathbf{p})>0$ or $\Delta f_{i}(\mathbf{p})<0$ (or both hold). Similar to the reason mentioned in the previous case, $\Delta \mathcal{E}_{i}(\mathbf{p})>$ $0 \Rightarrow \Delta \mathcal{E}_{i}^{d}(\mathbf{p})>0$. If $j$ is connected to $i$ (before and after the power level change), $\Delta \mathcal{E}_{i}^{d}(\mathbf{p})>0$ implies $\mathcal{E}_{i j}^{\min }(\mathbf{p})$ increases for at least one $j$ by definition, which in turn implies $\mathcal{E}_{k j}^{\min }(\mathbf{p})$ increases by virtue of Lemma 4, meaning that $\mathcal{E}_{k}^{d}(\mathbf{p})$ doesn't decrease. Thus, $\Delta \mathcal{E}_{k}(\mathbf{p})>$ $0 \Rightarrow \Delta u_{k}(\mathbf{p})<0$. In the latter case when $\Delta f_{i}(\mathbf{p})<$ $0 \Rightarrow \Delta f_{j}(\mathbf{p}) \leq 0$, meaning that $\Delta u_{j}(\mathbf{p}) \leq 0$. Thus, we have shown that $\Delta u_{i}(\mathbf{p})<0 \Rightarrow \Delta V(\mathbf{p})<0$.

Combining the above two cases, we have that $\operatorname{sgn}\left(\Delta u_{i}\right)=$ $\operatorname{sgn}(\Delta V), \forall i$, meaning that the game is an OPG.

One of the overaching consequences of being a potential game is the possible relationship between a potential function and a social welfare function. In the context of our TCG, the social welfare function is an energy-efficiency metric. Alternately, potential maximizing $\mathrm{NE}$ of the game can be interpreted as the optimal power assignment vectors, i.e., steady-state topologies that minimize the aggregate network energy consumption. This result builds upon the following lemma.

Lemma 7: The potential maximizing NE of the TCG preserves network connectivity.

Proof: We prove by contradiction. Let $\mathbf{p}^{*}$ be a potential maximizer and $\mathbf{p}$ any other $\mathrm{NE}$ that induces a connected network. Because $V\left(\mathbf{p}^{*}\right)>V(\mathbf{p})$, we have $\sum_{i}\left[\alpha_{i} f_{i}\left(\mathbf{p}^{*}\right)-\mathcal{E}_{i}\left(\mathbf{p}^{*}\right)\right]>\sum_{i}\left[\alpha_{i} f_{i}(\mathbf{p})-\mathcal{E}_{i}(\mathbf{p})\right]$. Since $\mathbf{p}^{*}$ results in a network that is not connected, $f_{i}\left(\mathbf{p}^{*}\right)=k_{i}<n-1$ and $f_{i}(\mathbf{p})=n-1$. Thus we obtain, $\sum_{i} \alpha_{i}\left(n-1-k_{i}\right)<$ $\sum_{i}\left[\mathcal{E}_{i}(\mathbf{p})-\mathcal{E}_{i}\left(\mathbf{p}^{*}\right)\right]$. Clearly, LHS is larger than $\mathcal{E}_{i}^{\max }$ and RHS is smaller than $\mathcal{E}_{i}^{\max }$. We thus obtain a contradiction.

Theorem 8: Minimum energy topologies $G_{\min }$ are the potential maximizing NE states.

Proof: From Lemma 7, the potential maximizing NE topology is always connected. Therefore, the potential function in the NE state becomes $V\left(\mathbf{p}^{*}\right)=M_{i} \cdot n(n-1)-\sum_{i} \mathcal{E}_{i}\left(\mathbf{p}^{*}\right)$. This implies,

$$
\mathbf{p}^{*}=\underset{\mathbf{p}}{\arg \min }\left\{\sum_{i}\left[\beta_{i} \mathcal{E}_{i}^{d}(\mathbf{p})+\kappa_{i} \mathcal{E}_{i}^{c}\left(p_{i}\right)\right]\right\}
$$

From the choice of $\beta_{i}$ and $\kappa_{i}$, it can be deduced that every node keeps all the minimum energy links and therefore minimizes $\mathcal{E}_{i}$. Thus, $\mathbf{p}^{\text {min }}$ satisifies (14). Hence, the minimum energy topologies maximize the potential function.
Through potential game formulation we have established the existence of at least one NE-the potential maximizer. When multiple NE exist, the task of eliminating the undesirable ones can be non-trivial. Besides, when one considers the convergence properties of the game, the problem is further compounded when multiple NE exist. Assuming that the threshold powers $\omega_{i j}$ are all distinct for distinct node pairs, the NE for the TCG is unique. We establish this via the following theorem, noting that the minimum energy topology is uniquely determined when $\omega_{i j}$ 's are distinct.

Theorem 9: Every NE of the TCG $\Gamma$ is a minimum energy topology.

Proof: Suppose that the NE topology doesn't contain a minimum energy path. This means, either some node has removed one of its minimum energy link or has removed a link on a minimum energy path for some other node. The former case violates the rationality principle, because a node can only increase its total energy cost by removing a minimum energy link. In the latter case, again, rationality principle is violated due to Lemma 4. Thus, in every iteration of a selfish algorithm ${ }^{4}$, all minimum energy paths are preserved. Hence, every NE is a minimum energy topology.

Corollary 10: In NE, the power level of every node is at the minimum level required to maintain $G_{\min }$.

Proof: We prove by contradiction. Suppose $p_{i}^{*}$ in NE is not at the minimum required to maintain $G_{\text {min }}$. This means, node $i$ can further reduce its power without disconnecting any of the minimum energy connections (if it does, then Theorem 9 is violated). Therefore, the original state is not a NE and we obtain a contradiction. The NE is given by $p^{*}=\left\{\left(p_{1}^{*}, p_{2}^{*}, \ldots, p_{n}^{*}\right) \mid p_{i}^{*}=\arg \min _{p_{i} \geq p_{i}^{\min }} \kappa_{i} \mathcal{E}_{i}^{c}\left(p_{i}\right)\right\}$.

\section{B. A TC Algorithm}

A NE is a consistent predictor of the likely outcomes of a game. For the TCG, the NE states are stable, efficient and unique. Consequently, the task of constructing a selfish algorithm that will converge to the NE is greatly simplified. This algorithm specifies which actions are rational from a node's perspective, and thus are likely to be chosen, given the state of the network. Because the TCG is a potential game, a simple BR construct ensures convergence to the NE. For the ease of exposition, we assume that all nodes initialize their power levels to $p_{i}^{\max }$ at the start of the algorithm such that the induced topology is $G_{\max }$ (this assumption, however, is not necessary for the correctness of the algorithm). In a BR algorithm, nodes make selfish adaptations, revising their selections according to:

$$
p_{i}^{*}=\underset{p_{i} \in\left[0, p_{i}^{\max }\right]}{\arg \max } u_{i}(\mathbf{p})
$$

When each node executes (15), a BR dynamic evolves, with nodes taking turns in making their optimum selections. We

\footnotetext{
${ }^{4}$ Without loss of generality, we may assume all nodes initialize their power levels to $p_{i}^{\max }$, such that the induced topology $G_{\max }$ contains the minimum energy topology $G_{\min }$.
} 
assume that only one node makes a selection (in a roundrobin manner) at any given instant ${ }^{5}$. The BR dynamic defines an improvment sequence; because each node essentially has to optimize over a finite set of power level choices (one for each of its potential neighbor) to select its minimum power, the improvement sequence is finite and guaranteed to converge to NE [6]. In the next section, we analyze and characterize the topologies that emerge when nodes employ the greedy BR strategy.

\section{Simulation Results}

To determine the effectiveness of our model, we developed a simulation consisting of $|N|$ radios placed according to a uniform random distribution within a unit square. The power thresholds $\omega(i, j)$ required to close a link between nodes $i$ and $j$ were assumed to be equal to $d^{2}(i, j)$ (we choose a path loss exponent of 2 , although our basic conclusions remain the same for other channel models as well), where $d$ is the euclidean distance metric. Based on our system model, a node could transmit reliably to all neighbors within its transmission range (determined by the power level).

The initial topologies of the network are connected, meaning that there exists a directed path from every radio to every other radio. The initial power $p_{i}^{\max }$ was chosen such that the induced network was 1-connected with $90 \%$ probability, adjusting the value for finite networks (see [20] for this formula). We consider only the connected instances of $G_{\max }$ in our simulations. The packet forwarding levels were exogenously selected; for the ease of exposition, the forwarding levels were kept constant across all nodes. We varied the levels from 0.1 to 1 , in steps of 0.1. Similar results can be drawn for non-uniform forwarding levels across the nodes.

Each node implemented the BR algorithm (15), selecting power levels that maximized (11). Nodes were selected in a round robin manner to make their decisions in each iteration. Other nodes are made aware of this adaptation through control messages. The traditional Dijkstra's algorithm was modified to a minimum energy routing algorithm for selecting optimal paths between node pairs. The modification is needed because the energy expression in (8) is not strictly additive because of $q$ term in the denominator, i.e. $\mathcal{E}_{i k} \neq \mathcal{E}_{i j}+\mathcal{E}_{j k}$. However, Dijkstra's algorithm can still be applied in principle by storing and updating both the energy cost terms in (8) in every iteration of the minimum cost path computation.

We begin by evaluating the effect of packet forwarding levels on the emergent topologies. At low forwarding levels, the expected energy consumed by long routes would be high, owing to greater number of retransmissions. To circumvent the high forwarding cost, we would expect nodes to transmit at higher powers inorder to reduce the number of hops to reach a destination. Consequently, the resultant steady state topologies will be highly connected, with nodes densely connected by greater number of routes. Figure 2 illustrates this result for a 25

\footnotetext{
${ }^{5}$ This can be implemented by embedding a random timer within each node, which can then make its selection every time its timer expires.
}

node network by computing the path lengths of the resulting NE topologies, averaged over 1000 different instances, with nodes randomly placed at different locations in each case.

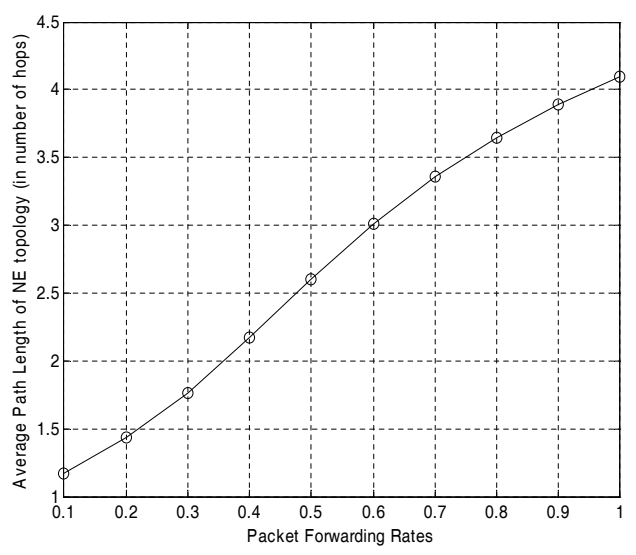

Fig. 2. Illustrating the impact of packet forwarding levels on the average path length of NE topologies in a 25 node network.

To determine the energy efficiency performance, we evaluated the total energy consumed by the NE topology obtained for various forward levels. At each level, the topologies were optimized to minimize the expected energy consumption. We compare each of the NE topology in Figure 3.

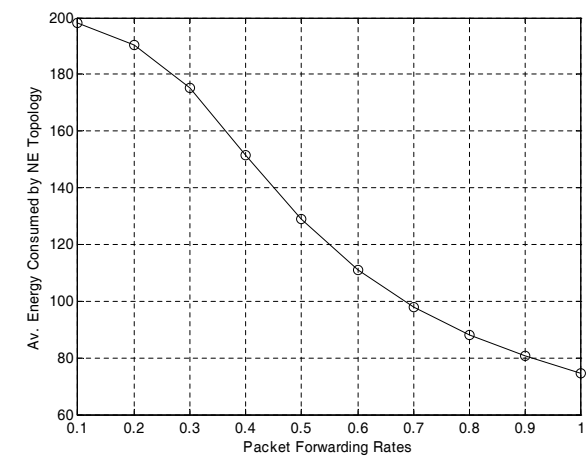

Fig. 3. Average energy consumed by a NE topology under various packet forwarding levels for a 25 node network.

A few insights can be drawn from Figure 3. The total energy consumed decreases montonically with increasing forwarding levels. This is because, at low forward levels, nodes use higher transmit power to offset the high cost of retransmission incurred by using the relay services. We see a gradual decrease in energy consumed because, with increasing forward levels, it costs less to transmit via a relay node than transmitting directly (even if retransmissions are involved). At high forward levels, the cost of direct transmissions dominates; therefore, it is more cost effective to transmit via intermediate nodes.

By quantifying the energy consumption in partially forwarding networks, Figure 3 also illuminates the performance gains achieved through cooperation in such networks. This result is particularly important when using reputation-based constructs 
to stimulate nodes to cooperate by forwarding packets for one another. Such schemes typically incur large overhead costs as they strive to steer the network towards cooperation. Choosing an optimum level of cooperation by balancing the cost performance tradeoff then becomes a design decision.

These results corroborate the well-established fact that it is better to relay messages when nodes are forwarding packets because of the exponential radio signal attenuation property. From a node viewpoint, we observe that if nodes act under the pretext that all destinations are equally likely, they benefit the most by forwarding. In some sense, if one could exchange packet forwarding services for real currency, nodes would incur minimum cost when they all forward packets for one another. These observations provide compelling evidence that aligning the individual selfish goals to the network objective is an important characteristic of a self-organizing network.

\section{BACKGROUND AND RELATED WORK}

Broadly, our work belongs to the body of research that addresses the impact of selfish node behavior on network performance. It is generally perceived that even if nodes act selfishly, some amount of cooperation is required to sustain an autonomous ad-hoc network (see [5] and references contained therein). The crux of the problem is how to stimulate the nodes to cooperate-by using reputation-based or pricingbased frameworks - when they are driven by self-interested objectives. The need for cooperation is a fundamental problem, which manifests in various forms at all layers of the protocol stack in a communication system [17].

The research efforts to address the problem of topology control in the presence of selfish nodes are fairly recent. Game theory and mechanism design are the commonly used approaches to address this problem. We now discuss the related work that uses these two approaches.

Eidenbenz et al. are the first to pose the TC problem as a non-cooperative game and study connectivity games [3]. Much of their work is devoted to the analysis of algorithmic complexity in finding a NE, when it exists, and deriving bounds on the price of anarchy. In [21], the authors formulate TC games as potential games. Potential games guarantee the existence of at least one NE. In addition, if the nodes employ any selfish algorithm to choose an appropriate power setting, convergence to these equilibria is also guaranteed.

In [3], existence of NE is not guaranteed. Furthermore, the authors do not provide energy-efficiency characteristics of the topologies that emerge. In [21], the steady state topologies that emerge are power-efficient but are not necessarily energyefficient. In contrast, we prove the existence as well as convergence results pertaining to globally energy-efficient $\mathrm{NE}$ topologies.

Mechanism design seeks to achieve global efficiency by aligning the selfish objectives of individual users with the socially desirable outcome. In the context of topology control, mechanism design is employed to provide the adequate incentives to individual users so that they maximize their objective function when the network minimizes total energy consumption, subject to connectivity constraints. This approach has been adopted in [19] and [18] by engineering a payment system that leads selfish nodes to forward packets for others. The utility function proposed in [18] requires that each node declare the per-edge price that it intends to charge in exchange for forwarding packets.

These studies assume upfront that incentivizing the nodes to cooperate and forward packets is the optimal policy, and then determine the appropriate payment structure that achieves this end goal. Our approach is different in that we make no assumptions that forwarding packets for one another is optimal. In our model, we allow the nodes to select and fix their forwarding levels, and then determine which topologies minimize the energy consumption, both from node and network perspectives. In the process, we also determine the optimal routing policy for nodes, from an energy minimization viewpoint.

While some similarities can certainly be drawn between our work and the other works in the literature, we believe that our approach of jointly considering packet forwarding and power control has not been addressed before in the context of topology control. In literature, most studies on energy efficiency in ad-hoc networks have dealt with these two issues separately.

\section{CONCLUSION}

Ad hoc networks are decentralized and their control is distributed to the independent and autonomous radios in the network. Such an "open" network philosophy leaves open the possibility that nodes can "game" the system in order to garner a greater share of network resources and achieve better performance. Selfish node behavior thus adds another layer of complexity to the traditional problems in distributed wireless networks in particular. Under this scenario, conventional protocols that are based on cooperation and truthful compliance can no longer be assumed to operate correctly. To effectively cope with these new dimensions in the problem, protocols may need to be redesigned.

We address the problem of topology control taking into account the presence of selfishly motivated nodes in the network. Specifically, we examine the impact of these nodes on the overall network energy efficiency. In multi-hop networks, nodes expend energy in sourcing as well as in forwarding packets. This may induce nodes to conserve their own energy and not adhere to a protocol that optimizes the overall network energy consumption. We cast this problem as a noncooperative game, where nodes attempt to select an optimum power level taking into account partial packet forwarding in the network; the objective of each node is to minimize its energy consumption while maintaining network connectivity.

We showed that the above TCG is a potential game. Through the potential game formulation we showed the uniqueness of the NE, which helps align the selfish objectives of the nodes with the overall network objective. In other words, when nodes selfishly minimize their energy expenditure, the network 
minimizes the aggregate energy consumption. We showed that a simple BR algorithm is sufficient to steer the nodes towards these globally efficient minimum energy topologies. Through simulations, we observed that when nodes are forwarding packets with high probabilities, the minimum energy topology is much sparser and consumes lesser energy than the NE topology containing low forwarding nodes. From an energy perspective, this result supports the philosophy of stimulating nodes to forward packets for one another in a decentralized, multi-hop network.

Our work presupposes that forwarding probabilities are selected exogenously. In future, we plan to address the energy minimization problem in a game-theoretic setting where the packet forwarding levels are also controlled selfishly. This will give rise to a multi-dimensional objective function that is a joint function of both transmit powers and packet forwarding levels.

\section{REFERENCES}

[1] C. E. Jones, K. M. Sivalingam, P. Agrawal, and J. C. Chen, "A Survey of Energy Efficient Network Protocols for Wireless Networks," Wireless Networks, vol. 7(4), pp. 343-358, Aug 2001.

[2] R. S. Komali and A. B. MacKenzie, "Distributed topology control in adhoc networks: A game theoretic perspective," in IEEE Consumer and Communication Networking Conference, vol. 1, pp. 563-568, January 2006.

[3] S. Eidenbenz, V. Kumar, and S. Zust, "Equilibria in topology control games for ad hoc networks," ACM/Kluwer Mobile Networks and Applications (MONET), vol. 11, no. 2, pp. 143-159, 2006.

[4] M. Felegyhazi, J.-P. Hubaux, and L. Buttyan, "Nash Equilibria of Packet Forwarding Strategies in Wireless Ad Hoc Networks," IEEE Transactions on Mobile Computing (TMC), vol. 5, no. 5, 2006.

[5] V. Srinivasan, P. Nuggehalli, C. Chiasserini, and R. Rao, "Cooperation in wireless ad hoc networks," in Proc. of IEEE INFOCOM, March 2003.

[6] D. Monderer and L. Shapley, "Potential games," Games and Economic Behavior, vol. 14, pp. 124-143, 1996.

[7] D. Fudenberg and J. Tirole, Game Theory. Cambridge: The MIT press, 1991.

[8] J. W. Friedman and C. Mezzetti, "Learning in games by random sampling," Journal of Economic Theory, vol. 98, pp. 55-84, 2001.

[9] V. Kawadia and P. R. Kumar, "Power control and clustering in ad hoc networks," in Proceedings of IEEE INFOCOM, vol. 1, pp. 459-469, 2003.

[10] E. S. Jung and N. H. Vaidya, "A power control MAC protocol for ad hoc networks," in Proc. ACM International Conference on Mobile Computing and Networking (MOBICOM), p. 3647, 2002.

[11] P. Karn, "MACA- a New Channel Access Method for Packet Radio," in Proc. 9th ARRL Computer Networking Conference, 1990.

[12] J. P. Monks, V. Bharghavan, and W. mei W. Hwu, "A Power Controlled Multiple Access Protocol for Wireless Packet Networks," in Proceedings of INFOCOM, April 2001.

[13] J. Gomez, A. T. Campbell, M. Naghshineh, and C. Bisdikian, "Conserving Transmission Power in Wireless Ad Hoc Networks," in ICNP'01, Nov 2001.

[14] S. Agarwal, S. Krishnamurthy, R. H. Katz, and S. K. Dao, "Distributed Power Control in Ad-hoc Wireless Networks," in Proc. of IEEE International Symposium on Personal, Indoor and Mobile Radio Communications(PIMRC), Oct 2001.

[15] X. Zhang and N. Maxemchuk, "A generalized energy consumption analysis in multihop wireless networks," in Proc. of IEEE WCNC, pp. 1476-1481, 2004.

[16] L. Li and J. Halpern, "A minimum-energy path-preserving topologycontrol algorithm," IEEE Transactions on Wireless Communications, vol. 3, no. 3, pp. 910-921, 2004.

[17] V. Srivastava, J. Neel, A. MacKenzie, R. Menon, J. Hicks, L. DaSilva, J. Reed, and R. Gilles, "Using game theory to analyze wireless ad hoc networks," IEEE Communications Surveys and Tutorials, Fourth Quarter 2005.
[18] P. Santi, S. Eidenbenz, and G. Resta, "A framework for incentive compatible topology control in non-cooperative wireless multi-hop networks," in DIWANS '06: Proceedings of the 2006 workshop on Dependability issues in wireless ad hoc networks and sensor networks, 2006.

[19] S. Yuen and B. Li, "Strategyproof mechanisms towards evolutionary topology formation in autonomous networks,", ACM/Kluwer Mobile Networks and Applications (MONET), Special Issue on Non-Cooperative Wireless Networking and Computing, Oct 2005.

[20] C. Bettstetter, "On the minimum node degree and connectivity of a wireless multihop network.," in In Proc. ACM Intern. Symp. on Mobile Ad Hoc Networking and Computing (MobiHoc), pp. 80-91, June 2002.

[21] R. S. Komali, A. B. MacKenzie, and R. P. Gilles, "Effect of selfish node behavior on efficient topology design," IEEE Transactions on Mobile Computing (TMC), To Appear. 